\title{
Induction chemotherapy followed by neoadjuvant chemoradiotherapy and surgery in locally advanced rectal cancer: preliminary results of a phase II study
}

\author{
Francesca De Felice ${ }^{1}$, Giancarlo D’Ambrosio², Daniela Musio ${ }^{1}$, Franco Iafrate ${ }^{1}$, \\ Ilaria Benevento ${ }^{1}$, Marco Marzo ${ }^{1}$, Marialaura Mancini ${ }^{1}$, Federica Urbano ${ }^{1}$, Marcella \\ Iannitti ${ }^{1}$, Francesco Marampon ${ }^{1}$, Nadia Bulzonetti ${ }^{1}$, Enrico Cortesi ${ }^{1,}{ }^{*}$ and Vincenzo \\ Tombolini, ${ }^{1, *}$ \\ ${ }^{1}$ Department of Radiological Sciences, Oncology and Pathology, Policlinico Umberto I, "Sapienza" University of Rome, Rome, \\ Italy \\ ${ }^{2}$ Department of General Surgery, Policlinico Umberto I, "Sapienza" University of Rome, Rome, Italy \\ "These authors have contributed equally to this work \\ Correspondence to: Francesca De Felice, email: fradefelice@hotmail.it \\ Keywords: rectal cancer; induction chemotherapy; mutation; chemoradiotherapy; complete response \\ Received: August 01, $2018 \quad$ Accepted: August 24, $2018 \quad$ Published: September 14, 2018 \\ Copyright: De Felice et al. This is an open-access article distributed under the terms of the Creative Commons Attribution License \\ 3.0 (CC BY 3.0), which permits unrestricted use, distribution, and reproduction in any medium, provided the original author and \\ source are credited.
}

\section{ABSTRACT}

Background and purpose: To report preliminary results of induction chemotherapy (IC) followed by neoadjuvant chemoradiotherapy (CRT) and surgery in locally advanced rectal cancer (LARC) patients.

Materials and methods: This is the preliminary evaluation of a phase II study. Patients with histologically proven rectal adenocarcinoma, stage II-III disease, who met the inclusion criteria, received induction FOLFOXIRI (5-FU, leucovorin, oxaliplatin and irinotecan) regimen in combination with targeted agents followed by CRT and surgery. Analysis of the first 8 patients was required to confirm the treatment feasibility before the accrual of $\mathbf{2 0}$ additional patients.

Results: The first 8 patients were evaluated. The median follow-up time was 23 months. There were no treatment-related deaths. Trimodality strategy was well tolerated with high compliance and a good level of toxicity. There were no evidence of febrile neutropenia and any grade 4 adverse events were recorded. Three patients had pathologic complete response (PCR) and 1 patient had a nearly pCR (ypT1 ypNO).

Conclusion: Preliminary results are encouraging. FOLFOXIRI regimen plus targeted agents followed by CRT and surgery seems a safe approach. Longer followup and higher number of patients are mandatory to confirm such findings.

\section{INTRODUCTION}

Despite validation of the trimodality treatment approach, including neoadjuvant 5-fluorouracil (5-FU)based chemoradiotherapy (CRT), surgery and adjuvant chemotherapy, distant metastasis remains a devastating issue of failure in locally advanced rectal cancer (LARC) management [1].

A recent meta-analysis concerning 3310 patients involved in four randomized trials showed a modest distant failure benefit $(\mathrm{OR}=0.76 ; 95 \% \mathrm{CI}, 0.60$ to $0.97 ; \mathrm{p}=0.03$ ) in adding oxaliplatin compared to standard 5-FU-based CRT [2]. But, definitive conclusions are still pending and the frequent development of distant metastasis in LARC continues to stimulate research community attention. The main consideration is the time window of approximately 4 months from neoadjuvant CRT to systemic chemotherapy which may potentially facilitate distant micrometastasis growth. Shifting systemic chemotherapy as initial approach into the trimodality treatment paradigm could 
Table 1: Patient characteristics

\begin{tabular}{lc}
\hline Characteristic & $\mathbf{n}(\mathbf{\%})$ \\
\hline Age (years) & $66(44-70)$ \\
median (range) & \\
Gender & $5(62.5)$ \\
Male & $3(37.5)$ \\
Female & \\
Smoke & $6(75)$ \\
Yes & $2(25)$ \\
No & \\
Comorbidity & $5(62.5)$ \\
none & $3(37.5)$ \\
Arterial hypertension & \\
Clinical tumor stage (T) & 0 \\
T1-2 & $3(37.5)$ \\
T3 & $5(62.5)$ \\
T4 & \\
Clinical nodal stage (N) & 0 \\
N0 & $2(25)$ \\
N1 & $6(75)$ \\
N2 & \\
Location from anal verge & cm \\
6 - 8 cm & $4(50)$ \\
\hline cm & $4(50)$ \\
\hline
\end{tabular}

represent a tangible option to target micrometastasis. Compared with postoperative CAPOX (capecitabine and oxaliplatin) regimen, induction CAPOX before neoadjuvant CRT achieved a more favorable compliance and toxicity profiles with similar pathologic complete response (pCR) rate, in several retrospective analysis and phase II randomized trials [3-4]. But these studies were not powered to draw any conclusions on survival outcomes. Therefore the risk of systemic spread remains a challenge.

Over the past decade, literature data reflects also a growing interest in defining a more effective systemic cytotoxic therapy. Year by year, Ras (KRas and NRas) and BRAF mutation status detection has become a crucial prognostic and predictive factor in colorectal patients [56]. Nowadays, the national comprehensive cancer network (NCCN) recommends tumor tissue genotyped for RasBRAF mutations in suspected or metastatic rectal cancers in order to tailor therapy and to confer - if any - benefit to patients [1].
Based on NCCN rectal guidelines and on the efficacy of the FOLFOXIRI (5-FU, leucovorin, oxaliplatin and irinotecan) regimen in combination with targeted agents demonstrated in wild-type KRas colorectal cancer patients $[1,7]$, we tested the utility of induction chemotherapy (IC) preceding neoadjuvant CRT and surgery resection in LARC. We report the preliminary results of a phase II study in order to provide an early opportunity to evaluate the efficacy of this new treatment sequence.

\section{RESULTS}

\section{Patient characteristics}

The first 8 patients were accrued between October 2015 and September 2016. Table 1 lists the demographics of the study population. In total, 5 patients were classified as cT4 and 4 patients had tumor in the low rectum. There was only 1 patients with wild-type Ras-BRAF tumor 
Table 2: Acute toxicity in preoperative phase

\begin{tabular}{|c|c|c|c|c|c|c|}
\hline \multirow{2}{*}{ Acute toxicity } & \multicolumn{2}{|c|}{ IC } & \multicolumn{2}{|c|}{ Neoadjuvant CRT } & \multicolumn{2}{|c|}{ Global } \\
\hline & G1-2 (\%) & G3-4 (\%) & G1-2 (\%) & G3-4 (\%) & G1-2 (\%) & G3-4 (\%) \\
\hline \multicolumn{7}{|l|}{ Allergy immunology } \\
\hline Allergic reaction hypersensitivity & - & - & - & - & - & - \\
\hline \multicolumn{7}{|l|}{ Constitutional symptoms } \\
\hline Fatigue & $4(50)$ & - & $3(37.5)$ & - & $7(87.5)$ & - \\
\hline Fever & $1(12.5)$ & - & $3(37.5)$ & - & $4(50)$ & - \\
\hline Palpitation & - & - & - & - & - & - \\
\hline \multicolumn{7}{|l|}{ Dermatology skin } \\
\hline Rash desquamation & $2(25)$ & - & $2(25)$ & - & $4(50)$ & - \\
\hline Radiation dermatitis & - & - & $2(25)$ & - & $2(25)$ & - \\
\hline \multicolumn{7}{|l|}{ Gastrointestinal } \\
\hline Disgeusia & $2(25)$ & - & - & - & $2(25)$ & - \\
\hline Oral mucositis & - & $1(12.5)$ & - & - & - & $1(12.5)$ \\
\hline Constipation & $1(12.5)$ & - & $2(25)$ & - & $3(37.5)$ & - \\
\hline Diarrhoea & $2(25)$ & - & $1(12.5)$ & - & $3(37.5)$ & - \\
\hline Nausea & $4(50)$ & - & - & - & $4(50)$ & - \\
\hline Vomiting & - & - & - & - & - & - \\
\hline Proctitis & - & - & $2(25)$ & - & $2(25)$ & - \\
\hline Haemorrhage rectum & $2(25)$ & - & - & - & $2(25)$ & - \\
\hline \multicolumn{7}{|l|}{ Metabolism disorders } \\
\hline Hyperkalemia & - & $1(12.5)$ & - & - & - & $1(12.5)$ \\
\hline ALT/AST increased & $2(25)$ & - & - & - & $2(25)$ & - \\
\hline \multicolumn{7}{|l|}{ Neurology } \\
\hline Neuropahy: sensory & $4(50)$ & - & $2(25)$ & - & $6(75)$ & - \\
\hline \multicolumn{7}{|l|}{ Pain } \\
\hline Abdominal pain or cramping & - & - & - & - & - & \\
\hline \multicolumn{7}{|l|}{ Renal genitourinary } \\
\hline Dysuria & - & - & $1(12.5)$ & - & $1(12.5)$ & - \\
\hline Urinary frequency & - & - & $1(12.5)$ & - & $1(12.5)$ & - \\
\hline \multicolumn{7}{|l|}{ Blood count } \\
\hline Neutropenia & $2(25)$ & $3(37.5)$ & - & - & $2(25)$ & $3(37.5)$ \\
\hline Haemoglobin & - & - & $1(12.5)$ & - & $1(12.5)$ & - \\
\hline
\end{tabular}

Abbreviations: IC: induction chemotherapy; CRT: chemoradiotherapy; G: grade; ALT/AST: alanine transaminase/ aspartate transaminase.

and he was treated with FOLFOXIRI regimen combined with panitumumab. The remainder $(n=7)$ received FOLFOXIRI regimen combined with bevacizumab.

\section{Induction chemotherapy compliance and toxicity}

Globally, 7 patients completed the planned cycles of IC. One patient underwent sudden cardiac death between the first and second cycle. The event was judged not related to study procedure. Patient history revealed arterial hypertension. In total, IC treatment was delayed because of toxic effect in one patient. Overall, grade 2 peripheral neurotoxicity $(n=2)$ and grade 3 neutropenia $(n=3)$ were observed. There were no evidence of febrile neutropenia and any grade 4 adverse events were recorded. The only other grade 3 toxic effects observed were oral mucositis $(\mathrm{n}=1)$ and hyperkalemia $(\mathrm{n}=1)$. No patients experienced grade 3 diarrhea. 


\section{Neoadjuvant chemoradiotherapy compliance and toxicity}

In total, 7 patients completed the programmed neoadjuvant CRT. As mentioned previously, one patient died during IC because of unrelated treatment cancer conditions. All patients received the RT prescribed total dose and no treatment interruptions were recorded. Due to IC-related toxicity, 4 patients received standard 5-FUbased concomitant chemotherapy. No patients suspended chemotherapy definitely, and thus they received at least 5 cycles of 5-FU with/without oxaliplatin. Acute toxicity incidences during IC and during CRT are shown in Table 2. Interestingly, there were no severe acute complications during neoadjuvant CRT.

\section{Trimodality treatment efficacy}

After the end of CRT, four patients had their tumor shrinked more than $50 \%$ of the original measurement. Tumor size evaluation at diagnosis (cDim), post-IC (yDim), post CRT (yyDim) and detected in the operative specimen (pDim) is demonstrated in Figure 1. A clinical complete response was noted in one patient, whereas clinical tumor reevaluation was stable in 2 cases. Conservative surgery was performed in five patients and the remainder $(\mathrm{n}=2)$ had a Miles surgery due to closeness to the anal verge. In total, 3 patients had $\mathrm{pCR}$ and 1 patient had a nearly pCR (ypT1 ypN0). None had positive radial margins. No peri-operative complications occurred. The median follow-up period was 23 months (range 18-28). At the time of the analysis, there were no treatment-related deaths. The estimated 1-year overall survival was $87.5 \%$ (0.387 - 0.981) (Figure 2). Among the 7 patients alive there was no initial evidence of local recurrence disease. One patient developed a distant relapse to the liver six months after surgery.

\section{DISCUSSION}

In the first stage of this two-stage design, 3 of the first 8 patients had pathologic complete response (pCR), which allowed the recruitment of 20 additional patients for the second stage. Globally, IC was well tolerated without negative impact - in term of interruption or major toxicities - on subsequent neoadjuvant CRT and resection. IC was given for up to four two-week cycles. There were

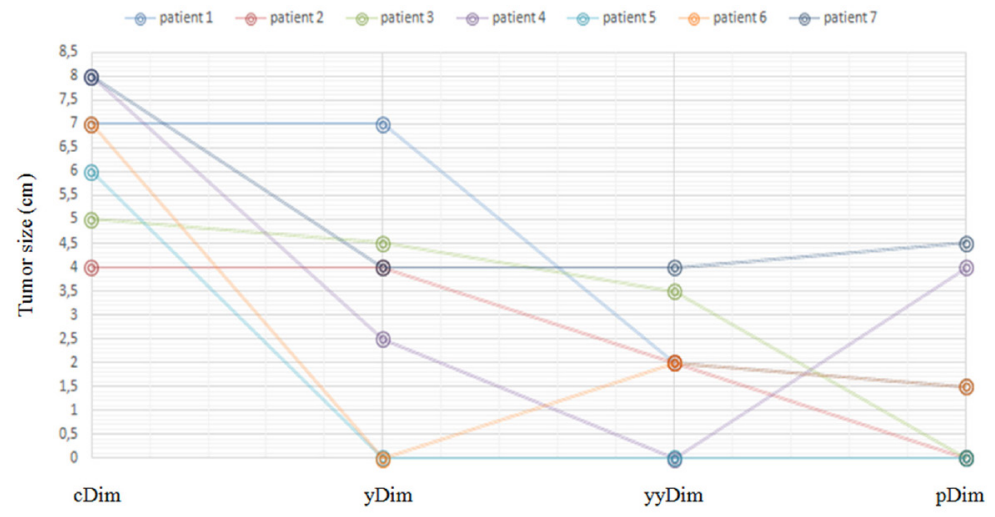

Figure 1: Tumor size evaluation. Tumor size at diagnosis (cDim), after induction chemotherapy (yDim), after neoadjuvant chemoradiotherapy (yyDim) and detected in the operative specimen (pDim).

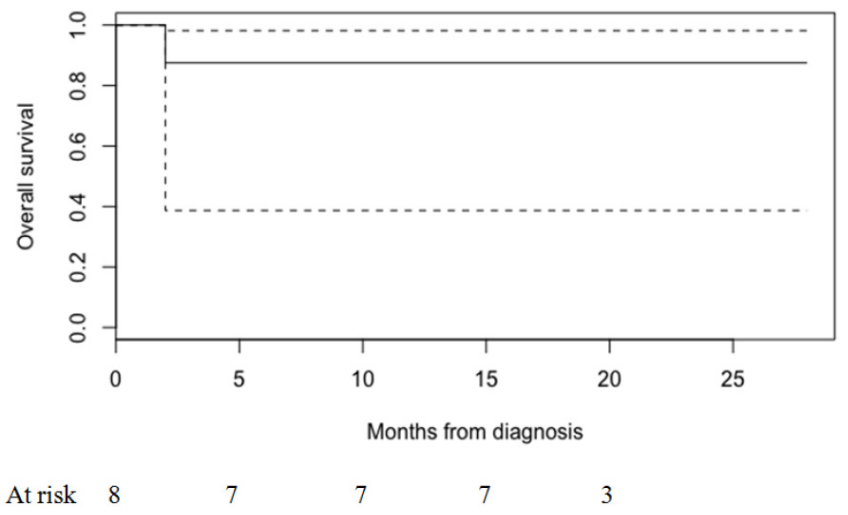

Figure 2: Overall survival. 
no grade 4 toxicities and no disease progression was seen during preoperative treatment phase. $\mathrm{R} 0$ resection was achieved in all patients who underwent surgery. To our knowledge, our experience represents the first series testing IC based on FOLFOXIRI regimen combined with targeted agents in LARC.

In the metastatic setting for colorectal cancer, FOLFOXIRI plus panitumumab or bevacizumab has shown promising clinical activity in Ras-BRAF wild-type patients [7,8]. The combination of triplet chemotherapy and panitumumab or bevacizumab has shown equivalent or superior safety profile, as well as significant improvement in tumor response compared with the available literature data for two cytotoxic drugs (a fluoropyrimidine plus either oxaliplatin or irinotecan) with or without biologics [7]. Therefore, it was rational for us to investigate FOLFOXIRI plus targeted agents as systemic chemotherapy in LARC. Moreover, considering that pCR has been shown to be an efficacious surrogate endpoint for survival outcomes in LARC [9-10], understanding the effect of chemotherapy intensification, using FOLFOXIRI plus targeted agents, in a preoperative setting could be essential to properly improve pCR rate. A phase II clinical trial was designed to provide an early opportunity to evaluate the efficacy of this new strategy treatment. It serves as a platform to highlight opportunities to change LARC approach drug, with the goal to improve survival outcomes. Preliminary data were encouraging, although second stage is mandatory to interpret final results and to appropriately translate them into clinical practice.

There are several considerations that should be made. Firstly, an earlier intervention with systemic treatment could facilitate full dose chemotherapy administration and tumor regression. It has been estimated that in more than $30 \%$ of LARC patients adjuvant chemotherapy is omitted or is dose reduced, increasing the risk of systemic failures [10]. Theoretical advantage of IC may allow improvement of oxygenation and higher intramural concentration of cytotoxic drugs to the primary tumor. Currently, Ras-BRAF became a high-priority target also in advanced rectal patients given the prevalence of mutations in rectal adenocarcinoma [11]. In our study, testing Ras-BRAF mutation status was paramount in driving therapeutic decisions. Cetuximab and panitumumab - two anti-epidermal growth factor receptor (EGFR)- targeted antibodies - are effective in wild-type disease [12-13]. Patients with known Ras mutations had virtually no chance of benefit from anti-EGFR agents and thus they were not treated with cetuximab and panitumumab. In fact, exposure to anti-EGFR agents toxicities would not be justified. Bevacizumab, an antiangiogenic agent, was administred in Ras-BRAF mutated patients, because its efficacy did not depend on the RasBRAF mutational status [11].

Secondly, our patient population, including all cases with cT3-4 disease and/or positive nodes but high-lying
cT3N0, is representative of a poor prognosis group. At diagnosis, all patients underwent pelvic DW-MRI and definition of poor prognosis disease was based on several parameters, including clinical stage, size, distance from anal merge, sphincter infiltration, potential mesorectal fascia involvement and extra-mural or venous invasion. Each of these parameters allowed an improved prediction of worse outcome, reinforcing the fact that an aggressive systemic strategy could show promising clinical benefits in this setting of patients [14-16]. Our main worry was that patients would be not able to tolerate subsequent CRT. Actually, all patients received neoadjuvant CRT without interruption or dose-limiting toxicity.

Lastly, in LARC, no data are available about the association between IC and survival outcomes after the introduction of targeted agents. FOLFOXIRI plus targeted agents was repeated every 14 days for up to 8 cycles (four two-week cycles). Short induction period (4 months) was selected in order to explore the benefit from intensification of neoadjuvant CRT in the sequential strategy.

At present, we were unable to address LF, DF and median OS due to the short interval follow-up. Moreover, patients cohort was too small to provide firm conclusions. As a results of a favorable pCR profile in the first stage of the two-stage design, the trial would continue to the second stage with the accrual of additional patients.

\section{MATERIALS AND METHODS}

This phase II study is a research project coordinates by Department of Radiological Sciences, Oncology and Pathology, Policlinico Umberto I "Sapienza" University of Rome and it was approved by the" Sapienza" University of Rome (number 88569-140). All patients provided written consent to participate. The preliminary evaluation of the first 8 patients was required to confirm the feasibility of the treatment before completing the enrollment of additional 20 patients.

\section{Patient selection}

Selection criteria included patients with newly diagnosed histologically proven rectal adenocarcinoma within $12 \mathrm{~cm}$ from the anal verge, staged T3-4 tumor and/ or positive lymph-nodes, without any evidence of distant metastases; age $\geq 18$ and $\leq 70$ years; performance status $\leq 2$; adequate renal, hepatic and bone marrow function. Patients with high-lying cT3N0 disease were not included. Patients were also excluded in case of synchronous tumors, cardiovascular disease, history of neurological or psychiatric disorders, previous pelvis radiotherapy, contraindication to MRI examination.

Clinical examinations, including complete medical history and careful physical examination, as well as digital rectal examination, were combined with radiologic imaging to assess the precise local $(\mathrm{T})$, regional nodal 
$(\mathrm{N})$, and distant (M) extent of the tumor. Radiologic imaging consisted of trans-rectal ultrasound, total body contrast-enhanced computed tomography (CT) and pelvic diffusion-weight magnetic resonance imaging (DW-MRI). Ras and BRAF mutational analyses was carried out on tumor biopsy.

\section{Study treatment}

All patients were treated with a multimodal treatment approach combining IC, followed by intensified neoadjuvant CRT and surgery.

IC consisted of four two-week cycles of the threedrug regimen of leucovorin-modulated 5-fluorouracil, irinotecan, and oxaliplatin (FOLFOXIRI). Modifications have been made to standard FOLFOX with goals of increasing efficacy. Moreover, based on Ras-BRAF status, biologic agents, including bevacizumab, panitumumab or cetuximab, were added. Only patients with wild-type KRas genes received treatment with cetuximab or panitumumab. Due to potentially severe toxicity, patients aged older than 70 years were excluded from the trial.

Therefore, IC consisted of four two-week cycles of the FOLFOXIRI regimen: irinotecan $165 \mathrm{mg} / \mathrm{m}^{2}$ day 1 , oxaliplatin $85 \mathrm{mg} / \mathrm{m}^{2}$ day 1 , leucovorin $200 \mathrm{mg} / \mathrm{m}^{2}$ day 1, fluorouracil $2400 \mathrm{mg} / \mathrm{m}^{2}$ 48-hour continuous infusion starting on day 1. In addition, cetuximab $\left(400 \mathrm{mg} / \mathrm{m}^{2}\right.$ first infusion, $250 \mathrm{mg} / \mathrm{m}^{2}$ thereafter) or panitumumab (6 $\mathrm{mg} / \mathrm{kg})$ or bevacizumab $(5 \mathrm{mg} / \mathrm{kg})$ were administered intravenously based on wild-type (cetuximab/ panitumemab) or mutated (bevacizumab) Ras-BRAF status. IC was left to the oncologists' discretion, because of individual variations in the patient conditions and medical circumstances.

Two weeks from the end of last IC cycle, the assessment of local clinical response was performed by pelvic DW-MRI. Independent of clinical response, long course of intensified CRT was started within four weeks after IC completion. The detailed CRT protocol has been described previously [17-18]. Radiation therapy was delivered with intensity modulate technique at a dose of 45 Gy $(1.8 \mathrm{~Gy} / \mathrm{fr})$ to the whole pelvis plus 5.4/9 Gy (1.8 Gy/ fr) to the tumor volume with 6 to $15 \mathrm{MV}$ energy photons. Concomitant chemotherapy consisted of OXP $\left(50 \mathrm{mg} / \mathrm{m}^{2}\right.$ on the first day of each week of RT) and 5-FU (200 mg/ $\mathrm{m}^{2} / 5$ daily continuous infusion).

Five weeks from the end of CRT, the assessment of local clinical response was performed by pelvic DW-MRI. Surgery was scheduled 7 to 9 weeks after the end of CRT treatment.

\section{Study end point and statistics}

Primary end point was the number of patients with pCR. pCR was defined as the absence of any residual tumor cells (ypT0) detected in the operative specimen, including the primary tumor area, the whole mesorectal fat, and the resected lymph nodes (ypN0).

Secondary end-points included toxicity and compliance with the regimen, tumor downstaging, R0 resection rate, surgical complications, sphincter preservation rate, local and distant failure rates, overall survival (OS). The assessment of local clinic response was performed by pelvic MRI and was based on investigatorreported measurements according to Response Evaluation Criteria in Solid Tumors (RECIST) guidelines [19]. Toxicity scoring was performed using the Common Terminology Criteria for Adverse Events, Version 4.0 [20]. OS was calculated in months from the date of diagnosis to the first event, including date of the last follow-up or death. Local failure (LF) and distant failure (DF) were defined as the time from diagnosis to local recurrence within the pelvis (LF) and distant metastasis (DF) occurrence. Standard descriptive statistics were used to evaluate the distribution of each factor. Continuous variables were presented as medians and interquartile range, and dichotomous variables were presented as counts and percentages.

The expected number of patients has been calculated according to the Simon's two-stage design. Based on four large randomized phase III trials [21-24], the projected $\mathrm{pCR}$ rate after standard preoperative treatment is $15 \%$ and an absolute $20 \%$ improvement in pCR rate is deemed clinically significant. With an $\alpha$ error of 0.05 and a power of $80 \%$, the planned study would proceed as followed: after a first stage of 8 patients, if three or more patients with a ypT0N0 tumor are observed, accrual of 20 additional patients will be achieved. If this condition is not met, the study will be stopped for futility. After the second step, if there are seven or more ypT0N0 tumor, it can be concluded that the rate of $\mathrm{pCR}$ is statistically significantly greater than that of literature.

Statistical analysis was carried out using R-Studio 0.98.1091 software.

\section{CONCLUSIONS}

Our preliminary data supported the continued patients accrual. IC based on FOLFOXIRI regimen combined with targeted agents followed by neoadjuvant CRT and surgical resection is manageable and well tolerated. This combination of therapies will hopefully provide meaningful benefit. Surely, longer follow-up and final results are mandatory to assess if this trimodality strategy consistently translate to improved overall survival and metastasis free survival in LARC patients.

\section{CONFLICTS OF INTEREST}

The authors declare that they have no competing interests. 


\section{REFERENCES}

1. National Comprehensive Cancer Network Guidelines. Rectal Cancer, Version 1. 2018. https://www.nccn.org.

2. De Felice F, Benevento I, Magnante AL, Musio D, Bulzonetti N, Caiazzo R, Tombolini V. Clinical benefit of adding oxaliplatin to standard neoadjuvant chemoradiotherapy in locally advanced rectal cancer: a meta-analysis : Oxaliplatin in neoadjuvant treatment for rectal cancer. BMC Cancer. 2017; 17:325. https://doi. org/10.1186/s12885-017-3323-4.

3. Fernández-Martos C, Pericay C, Aparicio J, Salud A, Safont M, Massuti B, Vera R, Escudero P, Maurel J, Marcuello E, Mengual JL, Saigi E, Estevan R, et al. Phase II, randomized study of concomitant chemoradiotherapy followed by surgery and adjuvant capecitabine plus oxaliplatin (CAPOX) compared with induction CAPOX followed by concomitant chemoradiotherapy and surgery in magnetic resonance imaging-defined, locally advanced rectal cancer: grupo cancer de recto 3 study. J Clin Oncol. 2010; 28:85965. https://doi.org/10.1200/JCO.2009.25.8541.

4. Chau I, Brown G, Cunningham D, Tait D, Wotherspoon A, Norman AR, Tebbutt N, Hill M, Ross PJ, Massey A, Oates J. Neoadjuvant capecitabine and oxaliplatin followed by synchronous chemoradiation and total mesorectal excision in magnetic resonance imaging-defined poor-risk rectal cancer. J Clin Oncol. 2006; 24:668-74. https://doi. org/10.1200/JCO.2005.04.4875.

5. Falcone A, Ricci S, Brunetti I, Pfanner E, Allegrini G, Barbara C, Crinò L, Benedetti G, Evangelista W, Fanchini L, Cortesi E, Picone V, Vitello S, et al, and Gruppo Oncologico Nord Ovest. Phase III trial of infusional fluorouracil, leucovorin, oxaliplatin, and irinotecan (FOLFOXIRI) compared with infusional fluorouracil, leucovorin, and irinotecan (FOLFIRI) as first-line treatment for metastatic colorectal cancer: the Gruppo Oncologico Nord Ovest. J Clin Oncol. 2007; 25:1670-76. https://doi. org/10.1200/JCO.2006.09.0928.

6. Tan $\mathrm{C}, \mathrm{Du} \mathrm{X}$. KRAS mutation testing in metastatic colorectal cancer. World J Gastroenterol. 2012; 18:5171-80.

7. Fornaro L, Lonardi S, Masi G, Loupakis F, Bergamo F, Salvatore L, Cremolini C, Schirripa M, Vivaldi C, Aprile G, Zaniboni A, Bracarda S, Fontanini G, et al. FOLFOXIRI in combination with panitumumab as first-line treatment in quadruple wild-type (KRAS, NRAS, HRAS, BRAF) metastatic colorectal cancer patients: a phase II trial by the Gruppo Oncologico Nord Ovest (GONO). Ann Oncol. 2013; 24:2062-67. https://doi.org/10.1093/annonc/mdt165.

8. Cremolini C, Loupakis F, Antoniotti C, Lupi C, Sensi E, Lonardi S, Mezi S, Tomasello G, Ronzoni M, Zaniboni A, Tonini G, Carlomagno C, Allegrini G, et al. FOLFOXIRI plus bevacizumab versus FOLFIRI plus bevacizumab as first-line treatment of patients with metastatic colorectal cancer: updated overall survival and molecular subgroup analyses of the open-label, phase 3 TRIBE study. Lancet Oncol. 2015; 16:1306-15. https://doi.org/10.1016/ S1470-2045(15)00122-9.

9. De Felice F, Izzo L, Musio D, Magnante AL, Bulzonetti N, Pugliese F, Izzo P, Di Cello P, Lucchetti P, Izzo S, Tombolini V. Clinical predictive factors of pathologic complete response in locally advanced rectal cancer. Oncotarget. 2016; 7:33374-80. https://doi.org/10.18632/ oncotarget.8133.

10. Schrag D. Neoadjuvant/Adjuvant Chemotherapy for Stage II/III Rectal Cancer. ASCO Annual Meeting. 2015.

11. Schmoll HJ, Van Cutsem E, Stein A, Valentini V, Glimelius B, Haustermans K, Nordlinger B, van de Velde CJ, Balmana J, Regula J, Nagtegaal ID, Beets-Tan RG, Arnold D, et al. ESMO Consensus Guidelines for management of patients with colon and rectal cancer. a personalized approach to clinical decision making. Ann Oncol. 2012; 23:2479-516. https://doi.org/10.1093/annonc/mds236.

12. Andreyev HJ, Norman AR, Cunningham D, Oates J, Dix BR, Iacopetta BJ, Young J, Walsh T, Ward R, Hawkins N, Beranek M, Jandik P, Benamouzig R, et al. Kirsten ras mutations in patients with colorectal cancer: the 'RASCAL II' study. Br J Cancer. 2001; 85:692-96. https://doi. org/10.1054/bjoc.2001.1964.

13. Lièvre A, Bachet JB, Le Corre D, Boige V, Landi B, Emile JF, Côté JF, Tomasic G, Penna C, Ducreux M, Rougier P, Penault-Llorca F, Laurent-Puig P. KRAS mutation status is predictive of response to cetuximab therapy in colorectal cancer. Cancer Res. 2006; 66:3992-95. https://doi. org/10.1158/0008-5472.CAN-06-0191.

14. Merkel S, Mansmann U, Siassi M, Papadopoulos T, Hohenberger W, Hermanek P. The prognostic inhomogeneity in pT3 rectal carcinomas. Int J Colorectal Dis. 2001; 16:298-304. https://doi.org/10.1007/ s003840100309.

15. Marijnen CA, Nagtegaal ID, Kapiteijn E, Kranenbarg EK, Noordijk EM, van Krieken JH, van de Velde CJ, Leer JW, and Cooperative investigators of the Dutch Colerectal Cancer Group. Radiotherapy does not compensate for positive resection margins in rectal cancer patients: report of a multicenter randomized trial. Int $\mathrm{J}$ Radiat Oncol Biol Phys. 2003; 55:1311-20. https://doi.org/10.1016/ S0360-3016(02)04291-8.

16. Marr R, Birbeck K, Garvican J, Macklin CP, Tiffin NJ, Parsons WJ, Dixon MF, Mapstone NP, Sebag-Montefiore D, Scott N, Johnston D, Sagar P, Finan P, Quirke P. The modern abdominoperineal excision: the next challenge after total mesorectal excision. Ann Surg. 2005; 242:74-82. https://doi.org/10.1097/01.sla.0000167926.60908.15.

17. De Felice F, Musio D, Magnante AL, Bulzonetti N, Benevento I, Caiazzo R, Tombolini V. Disease Control, Survival, and Toxicity Outcome After Intensified Neoadjuvant Chemoradiotherapy for Locally Advanced Rectal Cancer: A Single-Institution Experience. Clin 
Colorectal Cancer. 2016; 15:e17-22. https://doi. org/10.1016/j.clcc.2016.02.006.

18. Musio D, De Felice F, Bulzonetti N, Guarnaccia R, Caiazzo R, Bangrazi C, Raffetto N, Tombolini V. Neoadjuvantintensified treatment for rectal cancer: time to change? World J Gastroenterol. 2013; 19:3052-61. https://doi. org/10.3748/wjg.v19.i20.3052.

19. Eisenhauer EA, Therasse P, Bogaerts J, Schwartz LH, Sargent D, Ford R, Dancey J, Arbuck S, Gwyther S, Mooney M, Rubinstein L, Shankar L, Dodd L, et al. New response evaluation criteria in solid tumours: revised RECIST guideline (version 1.1). Eur J Cancer. 2009; 45:228-47. https://doi.org/10.1016/j.ejca.2008.10.026.

20. Cancer Therapy Evaluation Program. Common terminology criteria for adverse events, version 4.0. 2009. Available at: https://ctep.cancer.gov.

21. Gérard JP, Azria D, Gourgou-Bourgade S, Martel-Laffay I, Hennequin C, Etienne PL, Vendrely V, François E, de La Roche G, Bouché O, Mirabel X, Denis B, Mineur L, et al. Comparison of two neoadjuvant chemoradiotherapy regimens for locally advanced rectal cancer: results of the phase III trial ACCORD 12/0405-Prodige 2. J Clin Oncol. 2010; 28:1638-44. https://doi.org/10.1200/ JCO.2009.25.8376.

22. Rödel C, Liersch T, Becker H, Fietkau R, Hohenberger W, Hothorn T, Graeven U, Arnold D, Lang-Welzenbach
M, Raab HR, Sülberg H, Wittekind C, Potapov S, et al, and German Rectal Cancer Study Group. Preoperative chemoradiotherapy and postoperative chemotherapy with fluorouracil and oxaliplatin versus fluorouracil alone in locally advanced rectal cancer: initial results of the German CAO/ARO/AIO-04 randomised phase 3 trial. Lancet Oncol. 2012; 13:679-87. https://doi.org/10.1016/ S1470-2045(12)70187-0.

23. O'Connell MJ, Colangelo LH, Beart RW, Petrelli NJ, Allegra CJ, Sharif S, Pitot HC, Shields AF, Landry JC, Ryan DP, Parda DS, Mohiuddin M, Arora A, et al. Capecitabine and oxaliplatin in the preoperative multimodality treatment of rectal cancer: surgical end points from National Surgical Adjuvant Breast and Bowel Project trial R-04. J Clin Oncol. 2014; 32:1927-34. https://doi.org/10.1200/ JCO.2013.53.7753.

24. Aschele C, Cionini L, Lonardi S, Pinto C, Cordio S, Rosati G, Artale S, Tagliagambe A, Ambrosini G, Rosetti P, Bonetti A, Negru ME, Tronconi MC, et al. Primary tumor response to preoperative chemoradiation with or without oxaliplatin in locally advanced rectal cancer: pathologic results of the STAR-01 randomized phase III trial. J Clin Oncol. 2011; 29:2773-80. https://doi.org/10.1200/JCO.2010.34.4911. 